\title{
O Uso do Ultra-Som Intravascular na Cardiologia Intervencionista
}

\author{
Cesar Rocha Medeiros, Adriano Mendes Caixeta, Cláudia Mattos, Miguel Antônio Neves Rati \\ Rio de Janeiro, RJ
}

São abordados neste artigo os aspectos relevantes do uso do ultra-som intravascular na cardiologia intervencionista. Discute-se o aspecto ultra-sonográfico dos vasos coronarianos normais e acometidos por doença aterosclerótica, onde as análises qualitativa e quantitativa são salientadas como fatores primordiais na elaboração de uma estratégia terapêutica. Analisam-se as contribuições advindas com o uso do ultra-som intravascular para as intervenções coronarianas percutâneas, desde um melhor entendimento dos diversos mecanismos de desobstrução do lúmen arterial, até à utilização do ultra-som intravascular como guia para implante de endopróteses coronarianas. Os benefícios clínicos do uso do ultra-som intravascular são discutidos à luz de diversos estudos recentes, alguns desses randomizados e com indícios fortes de que o método influencia positivamente os prognósticos imediato e tardio das intervenções coronarianas percutâneas, tornando-se, portanto, forte aliado nos laboratórios de cardiologia intervencionista.

O estudo angiográfico dos vasos coronarianos é, desde a década de 50, o padrão para a detecção e para a caracterização anatômica da doença arterial coronariana. Porém, suas limitações são conhecidas e derivam do fato de a angiografia estudar, somente, $\mathrm{o}$ aspecto luminal do vaso (luminograma), o que pode ocultar detalhes sutis e importantes da artéria coronária. Sabe-se que a aterosclerose é uma doença predominantemente da parede arterial, havendo, portanto, a necessidade de um método que a avalie especificamente. A análise dos vasos coronarianos por intermédio do ultra-som intravascular permite informações detalhadas da parede arterial por analisar o aspecto interno do vaso em cortes tomográficos. A aquisição de imagens ultrasonográficas através de cateteres delgados e flexíveis apre-

Hospitais Barra D’Or e Copa D’Or - Rede D’Or - Rio de Janeiro, RJ Correspondência: Cesar Rocha Medeiros - Hospital Barra D’Or - Av. Ayrton Senna, 2541 - 22793-000 - Rio de Janeiro, RJ - E-mail: cmedei3@attglobal.net Recebido para publicação em 21/6/00 Aceito em $1 / 11 / 00$ senta-se como método adicional à angiografia, no que tange às abordagens diagnóstica e terapêutica da doença arterial coronariana. O estudo qualitativo e quantitativo das camadas da parede arterial sã e da placa de ateroma durante o procedimento diagnóstico ou terapêutico coronariano percutâneo é de grande valor clínico e experimental. Apesar dos benefícios clínicos advindos de seu uso em cardiologia intervencionista não serem ainda avalizados por grandes estudos randomizados, são crescentes as evidências de que o ultra-som intravascular pode ser importante aliado da terapêutica coronariana percutânea.

Características histológicas de uma artéria coronária normal- $\mathrm{O}$ aspecto histológico clássico, com três camadas concêntricas da parede arterial, pode também ser apreciado à avaliação da coronária pelo ultra-som intravascular $^{1-4}$ (fig. 1).

A íntima, camada mais interna da parede arterial, é compreendida entre o endotélio e a lâmina elástica interna, tendo ainda dentro de seus limites um subendotélio formado por células musculares lisas e fibroblastos dispostos em uma matriz de tecido conjuntivo. A detecção da camada íntima ao ultra-som intravascular depende de sua espessura, sendo a medida mínima de $160 \mu$ necessária para sua definição. A espessura da íntima aumenta com o avançar da idade, a despeito da ausência de lesões ateroscleróticas, alcançando o valor mínimo de $160 \mu$ em média aos trinta anos de idade nos homens; portanto, em indivíduos mais jovens, a análise da camada íntima ao ultra-som intravascular pode serprejudicada ${ }^{5}$.

A média consiste de várias camadas de células musculares lisas dispostas em matriz de pequena quantidade de fibras elásticas e colágeno, com espessura média de $200 \mu$ e separada da camada adventícia pela lâmina elástica externa. Ao ultra-som intravascular, a média apresenta-se como uma camada delgada e menos ecodensa do que a íntima e a adventícia, devido ao seu menor conteúdo de colágeno.

A adventícia, camada mais externa da parede arterial, tem espessura variável entre $300-500 \mu$ e é composta principalmente por tecido fibroso (colágeno e elastina), além de incorporar o vasa vasorum, nervos e vasos linfáticos. Ao 


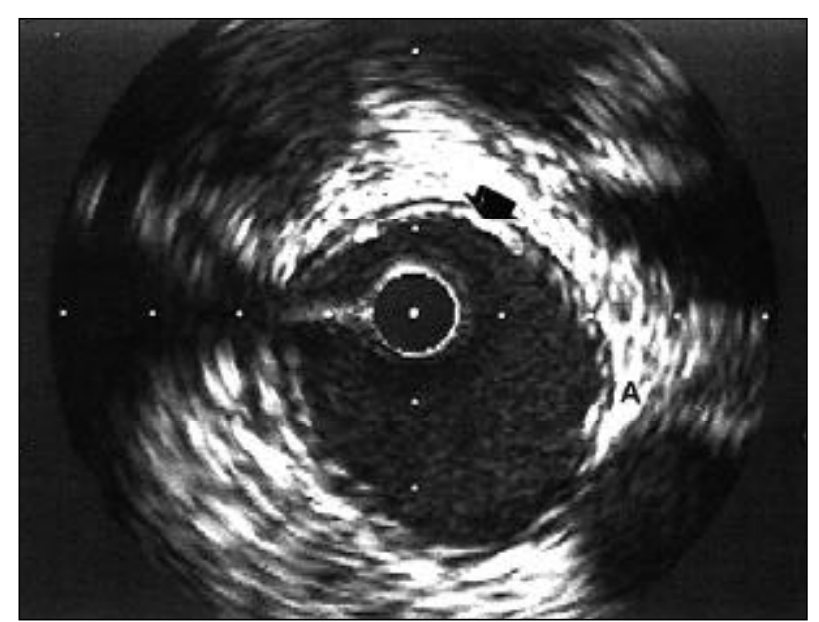

Fig. 1 - Aspecto ultra-sonográfico de uma artéria coronária normal. Observa-se o padrão característico das três camadas: A íntima, camada mais interna que se apresenta como um halo branco em contato com o lúmen (seta); a média, adjacente à camada íntima com o aspecto de um anel escuro; e a adventícia, camada mais externa, com aspecto de "casca de cebola" (A).

ultra-som intravascular, tem seus limites menos definidos do que os das outras camadas, confundindo-se, algumas vezes, com estruturas peri-vasculares.

Composição da placa aterosclerótica - A doença arterial coronariana é uma entidade de evolução longa e lenta, normalmente só detectada quando vem a provocar lesões luminais obstrutivas, alterando perfusão e causando sintomas. A coronariografia é incapaz de detectar fases precoces da doença arterial coronariana, pelo fato de estas caracterizarem-se como lesões não obstrutivas, não alterando, portanto, a silhueta angiográfica dos vasos. O ultra-som intravascular é um recurso útil na detecção de mudanças qualitativas que podem indicar desenvolvimento insidioso da aterosclerose coronariana. A mais precoce destas mudanças detectável ao ultra-som intravascular é o espessamento intimal presente de forma universal na doença arterial coronariana e mesmo no envelhecimento arterial normal. É proposta uma diferenciação empírica entre a placa aterosclerótica e o espessamento intimal fisiológico como sendo patológica a espessura do complexo íntima-média $>0,3 \mathrm{~mm}^{6}$.

Com a progressão da aterosclerose, podem-se identificar diferentes tipos de placa, de acordo com sua composição: 1) placas ditas "moles", com baixa ecodensidade (menor do que a da adventícia), são compostas por infiltração lipídica difusa e/ou células fibromusculares (fig. 2); 2) placas fibrosas com ecodensidade igual ou superior à da camada adventícia, que produzem ecos brilhantes e heterogêneos (fig. 3); e 3) placas calcificadas, que produzem reflexões brilhantes intensas e sombreamento acústico ${ }^{7,8}$ (fig. 4). Em uma série de pacientes submetidos à angioplastia, $82 \%$ das lesões exibiam algum grau de calcificação detectada ao ultra-som intravascular, sendo apenas $8 \%$ destas visíveis à angiografia ${ }^{3}$. A calcificação pode ser graduada de acordo com o arco da matriz fibrocálcica, sendo necessário um arco de $180^{\circ}$ para se adquirir uma massa de cálcio que possa ser identificada pela angiografia. O cálcio pode estar distribuído na placa de várias ma-

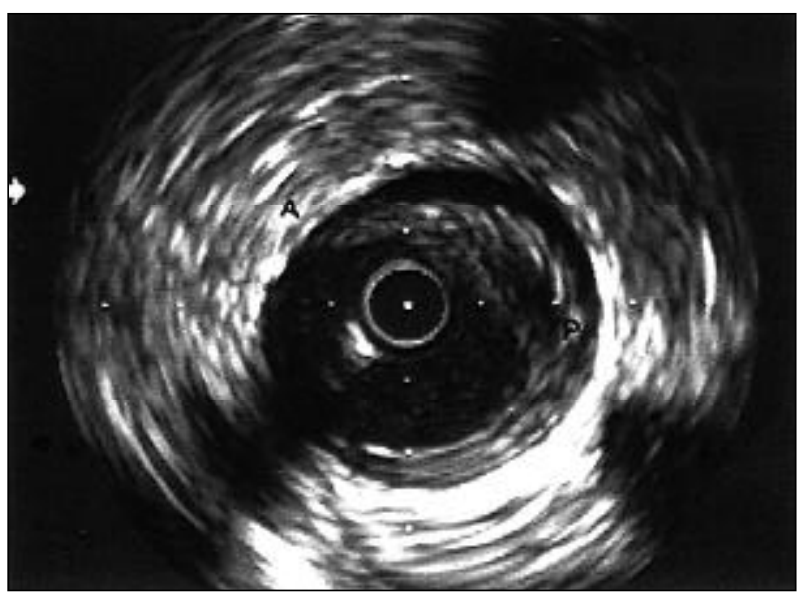

Fig. 2 - Placa aterosclerótica de conteúdo predominantemente lipídico. Observa-se ecodensidade menor da placa $(\mathrm{P})$ em relação à camada adventícia $(\mathrm{A})$.

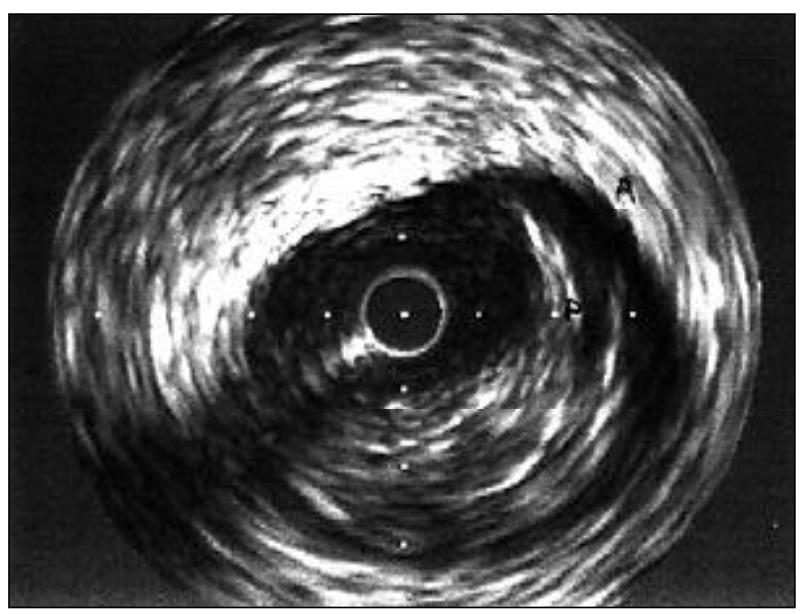

Fig. 3 - Placa aterosclerótica de conteúdo fibrótico. A ecodensidade da placa (P) assemelha-se à da camada adventícia (A).

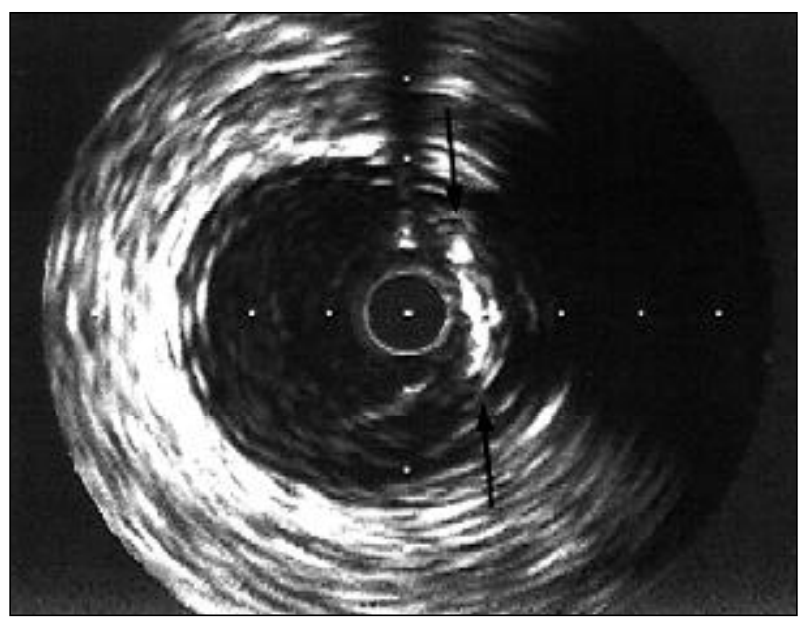

Fig. 4 - Placa calcificada. Observa-se um arco hiperdenso entre 2 e 4 h, produzindo sombreamento acústico (setas).

neiras: como um depósito profundo no limite íntima-média ( $15 \%$ dos casos), como um arco superficial na face luminal 
(50\%), uma combinação de ambas (35\%), ou como um foco inserido em uma placa fibrosa ${ }^{9,10}$. Ocasionalmente, áreas de deposição lipídica e degeneração necrótica aparecem como focos de baixa ecodensidade dentro de placas fibrosas ou cobertas por uma capa fibrótica (fig. 5). Placas ditas instáveis não raramente contém trombos aderidos à sua superfície, os quais têm como aspecto clássico ao ultra-som intravascular serem massas de aparência cintilante, com ecodensidade menor do que a da adventícia, que se movimentam de forma ondulada, diferentemente do movimento da artéria, e que eventualmente apresentam microcanais comunicando a placa ao lúmen do vaso. Apesar desses aspectos, a diferenciação entre um trombo e a placa "mole" pode, às vezes, ser impossível $^{11,12}$. Um estudo realizado in vitro com o ultra-som intravascular mostrou sensibilidade e especificidade na identificação de trombos de $57 \%$ e $91 \%$ respectivamente; ambas menores do que os índices obtidos com a angioscospia (sensibilidade e especificidade de $100 \%{ }^{13}$. Ainda como características de instabilidade de placa, podem ser detectadas ao ultra-som intravascular as rupturas e dissecções: a ruptura da placa é definida como uma descontinuidade radial da parede arterial, ou seja, perpendicular às camadas desta; já a dissecção caracteriza-se como uma lâmina paralela à parede arterial, comunicando-se com o lúmen através de um orifício ou fenda (fig. 6).

Análise quantitativa - $\mathrm{O}$ ultra-som intravascular é o método mais acurado para medidas do lúmen e do vaso coronário, permitindo o uso da planimetria para a análise destes parâmetros. As medidas mais utilizadas na prática clínica são expressas em área e diâmetro como as que se seguem: 1) área luminal: é medida traçando-se a borda da interface sangue/íntima. É o parâmetro mais adequado para quantificação ultra-sonográfica de lesões obstrutivas; 2) área total do vaso: é a área compreendida pelo limite média/adventícia, coincidindo com a posição da lâmina elástica externa. Esta medida não deve ser realizada, quando um arco de cálcio obscurecer mais do que 90 graus da circunferência do vaso. 3) área de placa: é a área que compreende a placa propriamente dita e a camada média, já que os limites entre estas du-

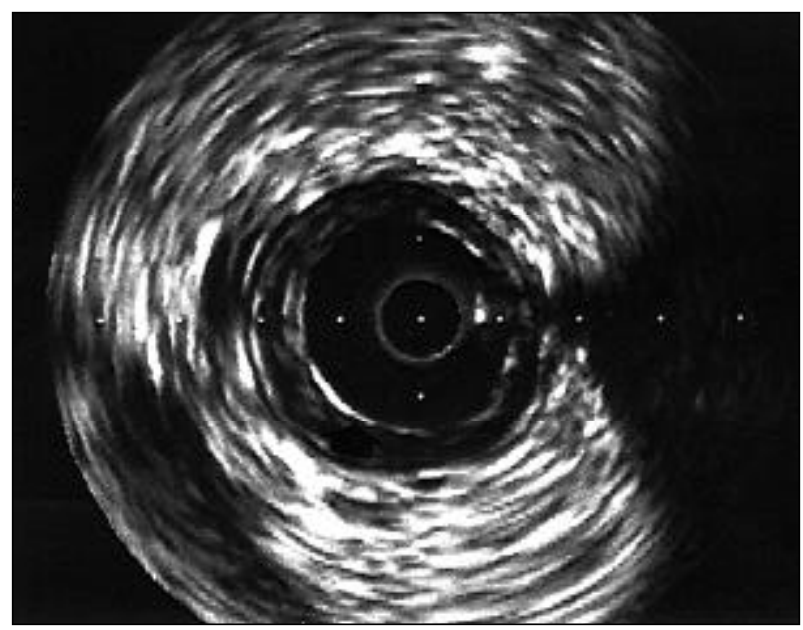

Fig. 5 - Placa mista. Observa-se material eminentemente lipídico contido por uma delgada capa fibrótica (seta).

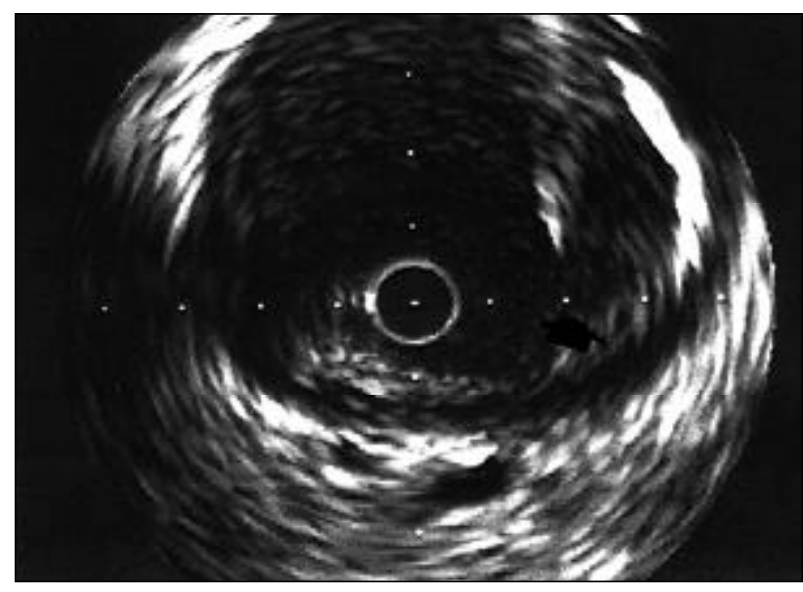

Fig. 6 - Dissecção da placa de ateroma. Nota-se uma fenda paralela às camadas da parede arterial entre 2 e 4 h. O orifício de entrada da dissecção é indicado pela seta.

as estruturas são, na maior parte das ocasiões, indistinguíveis. Esta medidaé, matematicamente, a diferença entre a área total do vaso e a área luminal. 4) área percentual de placa: expressa a percentagem da área total do vaso ocupada pela placa aterosclerótica. 5) diâmetro luminal mínimo: menor distância entre camadas íntimas diametralmente opostas. É utilizado freqüentemente para expressar o ponto de estenose mais acentuada. 6) diâmetro do vaso: é a distância entre camadas médias diametralmente opostas. Este diâmetro "média a média" nos segmentos de referência mostra-se fundamental na prática clínica diária, já que nesse baseia-se a escolha das dimensões do material utilizado em intervenção coronariana. Como diâmetros de referência proximal e distal, tomam-se os segmentos situados, no máximo, a 10mm da lesão, sendo que estes idealmente devem ser poupados de aterosclerose importante.

Apesar da utilização disseminada dos diâmetros luminais como medidas de expressão de gravidade de lesão e mesmo de prognóstico após intervenção coronariana, as medidas de área do vaso e do lúmen parecem correlacionarse de forma mais fidedigna às medidas funcionais de fluxo e pressão intracoronarianas.

As medidas obtidas pelo ultra-som intravascular, vieram confirmar as observações feitas no final da década passada acerca de um fenômeno conhecido como "remodelamento geométrico coronariano" ${ }^{15-16}$. Esse fenômeno, que aparentemente trata-se de um mecanismo compensatório intrínseco coronariano e surge em fases precoces da doença aterosclerótica, consiste na expansão circunferencial da artéria coronária no local de uma placa de ateroma (área total do vaso no local da lesão torna-se maior do que nas referências), na tentativa de acomodar o volume da placa e assim preservar o fluxo sangüíneo (remodelamento positivo) (fig. 7). A morfologia da placa parece exercer um papel importante no processo de remodelamento, pois este é mais precoce e mais intenso em placas excêntricas ${ }^{16}$. A extensão deste remodelamento, mesmo em se tratando de uma mesma artéria, pode ser extremamente variável, com segmentos exibindo remodelamento positivo, nenhum remodelamento, ou even- 


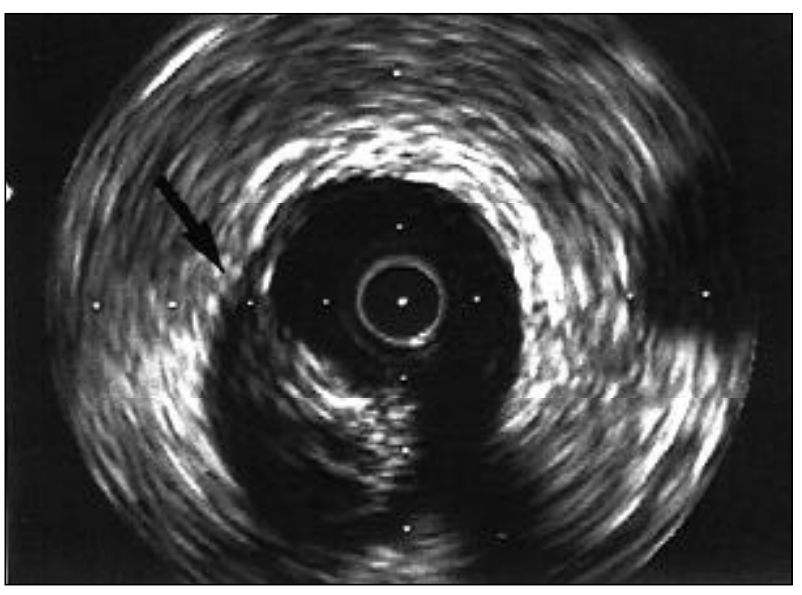

Fig. 7 - Remodelamento arterial positivo. Observa-se uma placa aterosclerótica excêntrica entre $6 \mathrm{e} 9 \mathrm{~h}$ causando um redimensionamento das camadas externas da parede arterial (seta).

tualmente até um remodelamento negativo (encolhimento). A razão de somente alguns segmentos arteriais apresentarem este mecanismo compensatório e o estágio da doença em que ele se torna insuficiente permanecem ainda por serem elucidados.

Aplicações diagnósticas do ultra-som - O ultra-som intravascular apresenta vantagens diagnósticas, particularmente nas situações em que há evidência de limitações à angiografia, como na detecção da doença angiograficamente silenciosa (lesões ostiais, síndrome X e vasculopatia do transplante), avaliação de lesões ambíguas, e avaliação de gravidade de estenose. Um estudo de Erbel e cols. demonstrou alterações ateroscleróticas ao ultra-som intravascular em $48 \%$ dos pacientes com doença coronariana clinicamente suspeita e coronariografia normal ${ }^{17}$. Dados como esses sugerem uma revisão e reclassificação de entidades, como a síndrome $\mathrm{X}$ ou a dor torácica sem alterações angiográficas. Na maioria das situações, o emprego do ultra-som intravascular ajuda na resolução de dilemas clínicos criados pela detecção angiográfica de lesões moderadas ou ambíguas, revelando-as não significativas ou mostrando estenoses acentuadas e/ou características de instabilidade da placa aterosclerótica. Algumas tentativas vêm sendo feitas no sentido de se criarem critérios para a definição da gravidade da lesão aterosclerótica ao ultra-som intravascular. Destaca-se o estabelecimento recente da área luminal mínima de $4,0 \mathrm{~mm}^{2}$, como valor absoluto de corte para o diagnóstico de estenose crítica em artérias nativas, o que foi validado ao demonstrar-se uma baixa taxa de eventos coronarianos originados por lesões cuja área luminal mínima excedesse esse valor ${ }^{18}$.

Aplicações do ultra-som em procedimentos terapêuticos - O crescimento nas indicações e o desenvolvimento de novas técnicas trouxe para a cardiologia intervencionista uma nova gama de desafios e possibilidades. A ampla disseminação dos métodos de intervenção coronariana percutânea exige que se garanta a conservação de sua segurança e o aumento de sua precisão, e o ultra-som intravascular parece ser uma importante ferramenta neste sentido.
A avaliação pré-procedimento do grau da lesão obstrutiva, bem como de sua morfologia e de suas dimensões de referência, é o passo inicial do planejamento estratégico na intervenção coronariana. O ultra-som intravascular oferece maior acurácia no dimensionamento coronariano, evidenciando diâmetros cerca de $10 \%$ maiores em relação à angiografia e correlacionando-se melhor com achados anatomopatológicos ${ }^{19,27}$. Além das dimensões, a análise morfológica é crucial para a escolha de uma eventual átero-ablação; como já salientado, o ultra-som intravascular é o método de escolha para a detecção de cálcio intracoronariano e definição de excentricidade da placa aterosclerótica, indicações de aterectomia rotacional e direcional, respectivamente. Em serviços com operadores experientes, o uso do ultra-som intravascular pré-procedimento chega a alterar o planejamento estratégico em até $40 \%$ dos procedimentos ${ }^{19}$.

Apesar do uso bastante difundido do cateter-balão para tratamento percutâneo de lesões obstrutivas coronarianas, o mecanismo pelo qual seu uso promove ganho luminal ainda é motivo de debate ${ }^{20}$. O ultra-som intravascular veio revelar que, aparentemente, os principais mecanismos de ganho luminal após angioplastia com balão são as rupturas e dissecções da placa aterosclerótica, principalmente quando estas contém cálcio. Estas rupturas e dissecções chegam a representar $72 \%$ do ganho luminal total em área ${ }^{21,22}$. Outro mecanismo potencial de ganho luminal pósangioplastia é a distensão da parede arterial, que parece ocorrer com maior frequiência ao se lidar com placas ditas moles; este mecanismo é definido ao se observar, após a angioplastia, uma área total do vaso no local da lesão alvo maior do que a observada no segmento de referência proximal ${ }^{23}$. O terceiro mecanismo, proposto por Dotter e Judkins ${ }^{24}$, sugere que haja uma compressão radial da placa, o que resultaria em um ganho luminal de cerca de $20 \%$; porém, análises recentes apontam para uma redistribuição axial da placa, ou seja, ocorre uma diminuição do volume da placa no local da lesão alvo; entretanto, há um aumento deste volume em porções mais proximais ou distais, sugerindo que o balão, na verdade, esprema o volume da placa em direção às bordas da lesão ${ }^{25,26}$.

A utilização do ultra-som intravascular permitiu ainda o desenvolvimento de técnicas de angioplastia mais agressivas, uma vez que este método freqüentemente permite um dimensionamento mais generoso dos vasos coronarianos, resultando no uso seguro de cateteres-balão maiores, com melhores resultados angiográficos imediatos e menor taxa de eventos clínicos a longo prazo ${ }^{27,28}$.

Além da definição dos mecanismos de ganho luminal e do melhor dimensionamento dos balões, o ultra-som intravascular auxilia na definição da forma do lúmen após angioplastia, já que esta, ao criar dissecções e rupturas, torna os contornos luminais irregulares com detalhes imperceptíveis à apreciação angiográfica. O ultra-som intravascular pósangioplastia serve ainda para revelar dissecções e/ou grandes volumes de placa não suspeitados à angiografia, achados que poderiam comprometer os resultados de um procedimento previamente definido comostent-like ${ }^{29}$. 
O ultra-som intravascular desempenhou um papel fundamental no entendimento e otimização do implante de stents intra-coronarianos. Nos primeiros anos de sua utilização, a complicação mais temida após o implante de stent coronariano era a trombose em seu interior, o que concorria para um elevado percentual de morbi-mortalidade pós-procedimento. Visando-se a redução desses fenômenos trombóticos, a anticoagulação com dicumarínicos era rotineiramente recomendada, trazendo consigo todos os riscos inerentes ao seu uso. As primeiras observações pelo ultra-som intravascular demonstraram que a técnica de implante de stent utilizada em grandes estudos como o STRESS e o BENESTENT $^{30,31}$ freqüentemente resultava em expansão inadequada e/ou má aposição das hastes do stent. Esta técnica, hoje conhecida como implante com baixa pressão, determinava áreas luminais intra-stent de somente cerca de 50 a 75\% da área esperada pela insuflação do cateter-balão em sua pressão nominal, a despeito de excelentes resultados angiográficos ${ }^{32-34}$.

Após correlacionar-se a subexpansão do stent ao risco de fenômenos trombóticos, um estudo clássico de Colombo e cols. ${ }^{35}$ descreveu uma técnica de implante guiada por ultra-som intravascular visando a expansão e aposição ótima dos stents, tornando desnecessário o uso de anticoagulação posterior. A técnica consistia no uso rotineiro de altas pressões de implante, tendo como objetivo alcançar uma área luminal maior do que $60 \%$ da média das áreas de referência proximal e distal; durante o estudo, entretanto, a definição de implante ótimo do stent foi modificada, considerando-se adequada a expansão onde a área intra-stent superasse à da referência distal. Nos pacientes em que a expansão ótima era atingida, preenchendo-se os critérios ultra-sonográficos, o uso dos dicumarínicos era considerado desnecessário e o tratamento proposto consistia em ácido acetilsalicílico e ticlopidina, resultando em taxas de trombose subaguda menores que $1 \%$ e de reestenose sintomática em seis meses de $13 \%$.

A partir desse estudo, o uso de anticoagulação oral foi abolido pela maioria dos serviços de cardiologia intervencionista em prol da antiagregação plaquetária, e o uso das altas pressões para implante de stents tornou-se rotina.

Considerando-se esses achados, começaram a surgir registros da técnica de implante com alta pressão sem a monitorização pelo ultra-som intravascular, obtendo-se uma incidência de trombose subaguda bastante semelhante à atingida, quando do uso do ultra-som intravascular como guia do procedimento, tornando seu uso de benefício duvidoso neste aspecto ${ }^{36,37}$. Este fenômeno talvez se deva à incidência ínfima dos fenômenos trombóticos após implante de stents nos grandes estudos sem o uso do ultra-som intravascular. Já no que tange à reestenose, a outra grande limitação no seguimento clínico após implante de stents, cada vez mais as evidências apontam para o benefício da aquisição de maiores áreas luminais após procedimento, obtidas de forma significativamente mais freqüente quando o ultrasom intravascular é utilizado; mesmo na era do implante com altas pressões, a monitorização com o ultra-som intra- vascular leva a um aumento médio da área luminal mínima intra-stent de cerca de $25 \%{ }^{38,39}$. Em nossa experiência, após implante de stents utilizando-se altas pressões de insuflação, mesmo com a obtenção de resultado angiográfico adequado, houve necessidade de nova expansão baseada nos achados do ultra-som intravascular em 59\% dos procedimentos, o que determinou ganho luminal adicional de $30 \%$ $(p=0,046)^{40}$. Pelo fato de a reestenose intra-stent ser predominantemente determinada pela quantidade de hiperplasia intimal, assume-se que lúmens maiores podem acomodar mais tecido proliferativo sem que isto cause comprometimento de fluxo ${ }^{41}$. A significância clínica destas considerações ainda está pendente, dependendo de grandes estudos randomizados para sua ratificação, porém, existem fortes tendências apontando para a redução de reestenose e revascularização do vaso alvo a longo prazo $(6,3 \%$ e $35 \%$, respectivamente) em estudos como SIPS, RESIST, MUSIC, CRUISE, OPTICUS, AVID ${ }^{42-47}$.

A crescente experiência advinda da utilização em larga escala do ultra-som intravascular para obtenção de grandes áreas luminais intra-stent, fez com que fossem modificados os critérios de ótimo implante de stents, como propôs o estudo MUSIC ${ }^{44}$. Esse estudo sugere os seguintes objetivos ao ultra-som intravascular: a) aposição completa das hastes do stent em todo o seu comprimento; b) área luminal mínima intra-stent maior ou igual a $90 \%$ da média das áreas de referência proximal e distal, ou maior ou igual a 100\% da área da menor referência; este critério é modificado ( $80 \%$ da média das áreas de referência ou $90 \%$ da menor área de referência) se a área luminal mínima intra-stent for maior do que $9 \mathrm{~mm}^{2} ; \mathrm{c}$ ) expansão simétrica do stent definida pela relação entre os diâmetros luminais mínimo/máximo (maior ou igual a 0,7 ). Embora o preenchimento desses critérios só seja atingido em cerca de $60 \%$ dos procedimentos ${ }^{43}$, esses parâmetros devem ser considerados como objetivo ideal da intervenção, já que este mesmo estudo observou taxas de revascularização de vaso alvo de apenas $3 \%$ ao se atingir área luminal mínima intra-stent de $9 \mathrm{~mm}^{244}$. A aquisição de área luminal intra-stent maior do que $9 \mathrm{~mm}^{2}$ somente é possível em vasos com o diâmetro de referência maior do que $3,5 \mathrm{~mm}$, em nossa experiência, o que por si já consiste em fator preditor de evolução favorável após implante de stent. Já o estudo $\mathrm{AVID}^{47}$, cujo seguimento clínico tardio foi apresentado no congresso de 1999 da American Heart Association, demonstrou grandes benefícios no implante de stents guiado pelo ultra-som intravascular no que se refere à taxa de nova revascularização do vaso alvo, principalmente em vasos menores do que $3,25 \mathrm{~mm}$ de diâmetro, onde a necessidade de nova revascularização foi de $8,6 \%$ no grupo do ultra-som intravascular contra $17,2 \%$ no grupo onde este não foi utilizado; e em pontes de safena, onde a taxa de nova revascularização foi de $3,4 \%$ contra $14,4 \%$, favorecendo o grupo onde o ultra-som intravascular foi empregado.

Quanto à aterectomia rotacional, o ultra-som intravascular veio a confirmar o mecanismo de ablação diferencial promovido por este método, ou seja, o material inelástico 
(cálcio) sofre abrasão pela oliva, enquanto os tecidos elásticos normais são apenas afastados desta, não sofrendo alterações significativas ${ }^{48}$.

Utilizando-se o ultra-som intravascular, existe ainda a possibilidade do dimensionamento da oliva em relação aos diâmetros da artéria, visando-se a relação ideal de 0,8:1, o que é extremamente difícil levando-se em conta apenas os dados angiográficos, já que freqüentemente a angiografia subestima estes diâmetros, principalmente em vasos com aterosclerose difusa, comumente tratados com aterectomia rotacional $^{48,49}$.

A aterectomia direcional também vem sendo objeto de estudo com ultra-som intravascular. Utilizando-se imagens ultra-sonográficas, pequenos estudos revelaram que a remoção da placa parece desempenhar um papel um pouco mais importante do que a distensão da parede arterial no ganho luminal após a aterectomia direcional ${ }^{50,51}$.

O ultra-som intravascular passou a ser um método auxiliar importante para a aterectomia direcional na seleção das lesões a serem tratadas, ao revelar a quantidade e a localização de cálcio na coronária, já que é sabido que a presença de cálcio superficial é o principal preditor de insucesso ao se usar este método ${ }^{52,53}$.

Um crescente número de estudos vem sendo realizado guiando-se o aterótomo pelo ultra-som intravascular e adquirindo-se lúmens iniciais maiores e menores taxas de reestenose ${ }^{52,53}$. O registro multicêntrico OARS ${ }^{54}$ mostrou uma redução aguda de $63,5 \%$ para $7 \%$ no diâmetro de estenose das lesões tratadas, com taxas de reestenose angiográfica em seis meses de $28,9 \%$, tendo como seu maior preditor um diâmetro luminal pequeno pós-procedimento; números portanto bem menores do que os 46 a $50 \%$ de reestenose gerados pela aterectomia direcional sem o uso do ultra-som intravascular, como demonstrados nos estudos CAVEAT e CCAT ${ }^{55,56}$.

O ultra-som intravascular e os mecanismos de reestenose - Apesar de todos os avanços em técnica, equipamento e farmacologia adjunta, a reestenose permanece como o principal limitante às intervenções coronarianas percutâneas. O ultra-som intravascular veio oferecer novas perspectivas para o entendimento dos possíveis mecanismos de reestenose após a utilização das diversas técnicas intervencionistas.

O ultra-som intravascular permitiu-nos estabelecer alguns fatores preditores de reestenose, tais como: a aquisição de uma área luminal absoluta e/ou relativamente pequena após o procedimento, uma área pequena em termos absolutos do segmento de referência, má aposição e expansão dostent, e um grande volume residual de placa aterosclerótica ${ }^{57}$.
Estudos com ultra-som intravascular em coronárias submetidas à angioplastia por cateter-balão realizados por recorrência de sintomas e/ou por força de protocolos de pesquisa, revelam que o encolhimento da área total do vaso, fenômeno conhecido como remodelamento geométrico negativo, chega a responder por até $70 \%$ da perda luminal vascular, enquanto que a proliferação neointimal é responsável por cerca de $23 \%$ dessa perda ${ }^{58}$.

Ao contrário do mecanismo de reestenose responsável pela perda luminal após a utilização do cateter-balão e dos equipamentos de aterectomia, que parece ser um misto de remodelamento negativo e proliferação neointimal, a reestenose intra-stent é causada primordialmente pela proliferação neointimal ${ }^{58}$. A área compreendida pelas hastes do stent permanece praticamente inalterada, resistindo ao processo de remodelamento da parede arterial, enquanto que o crescimento intimal correlaciona-se de forma diretamente proporcional à perda luminal ocorrida meses após o implante do stent, ao se reestudar o vaso com o auxílio do ultra-som intravascular ${ }^{59}$.

No que tange ao tratamento da lesão reestenótica, o ultra-som intravascular oferece uma idéia mais precisa das medidas e características do vaso e da hiperplasia intimal, facilitando a escolha e dimensionamento do material. Freqüentemente, o ultra-som intravascular flagra uma expansão inadequada de stent, o que pode ser tratado com o redimensionamento do cateter-balão ${ }^{60}$.

Apesar da inestimável contribuição do ultra-som intravascular em todos estes aspectos, a prevenção e o tratamento da reestenose após as intervenções coronarianas percutâneas permanecem pouco esclarecidas, necessitando de grandes estudos randomizados para seu melhor entendimento.

Perspectivas - Para os próximos anos, já se podem vislumbrar alguns avanços na técnica e nos materiais utilizados para a aquisição de imagens em ultra-som intravascular. A reconstrução tridimensional das imagens tem sido proposta como uma forma de facilitar o entendimento da relação espacial entre as estruturas, porém, devido a algumas limitações técnicas, sua utilização atual limita-se a pesquisas de pequena escala ${ }^{61}$. A combinação do transdutor de ultrasom intravascular a um dispositivo terapêutico (balão de angioplastia, aterótomo direcional ou stent) no mesmo cateter surgiu como uma idéia bastante atraente, carecendo, ainda, de ajustes técnicos que permitam sua incorporação à prática clínica ${ }^{62}$. Com relação à braquiterapia, o ultra-som intravascular é atualmente o método padrão para dimensionamento de vasos e lesões, o que tem implicações diretas no estabelecimento da dosagem do radioisótopo a ser utilizada. 


\section{Referências}

1. Gussenhoven EJ, Essed CE, Lancee CT, et al. Arterial wall characteristics determined by intravascular ultrasound imaging: An in vivo study. J Am Coll Cardiol 1989; 14: 947-52

2. Nishimura RA, Edwards WD, Warner CA, et al. Intravascular ultrasound imaging: In vitro validation and pathologic correlation. J Am Coll Cardiol 1990; 16: 145-54.

3. Tobis JM, Mallery J, Mahon D, et al. Intravascular ultrasound imaging of human coronary arteries in vivo. Analysis of tissue characterization with comparison to in vitro histological specimens. Circulation 1991; 83: 913-26.

4. Siegel RJ, Chae JS, Maurer G, Berlin M, Fishbein MC. Histopathologic correlation of the three-layered intravascular ultrasound appearance of normal adult human muscular arteries. Am Heart J 1993; 126: 872-8.

5. Fitzgerald PJ, St Goar FG, Connolly AJ, et al. Intravascular ultrasound imaging of coronary arteries. Is three layers the norm? Circulation 1992; 86: 154-8.

6. Velican D, Velican C. Comparative study on age related changes and atherosclerosis involvment of the coronary arteries of male and female subjects up to 40 years of age. Atherosclerosis 1981; 38: 39-50.

7. Di Mario C, The SHK, Madretsma S, et al. Detection and characterization of vascular lesions by intravascular ultrasound: an in vitro study correlated with histology. J Am Soc Echocardiogr 1992; 5: 135-46.

8. Bartorelli AL, Potkin BN, Almagor Y, Keren G, Roberts WC, Leon MB. Plaque characterization of atherosclerotic coronary arteries by intravascular ultrasound. Echocardiography 1990; 7: 389-95.

9. Farb A, Virmani R, Atkinson JB, Kolodgie FD. Plaque morphology and pathologic changes in arteries from patients dying after coronary balloon angioplasty. J Am Coll Cardiol 1990; 16: 1421

10. Mintz GS, Douek P, Pichard AD, et al. Target lesion calcification in coronary artery disease: an intravascular ultrasound study. J Am Coll Cardiol 1992; 20 1149-55.

11. Kearney P, Koch L, Ge J, Gorge G, Erbel R. Differences in morphology of stable and unstable coronary lesions and their impact on the mechanism of angioplasty. An in vivo study with IVUS. Eur Heart J 1996; 17: 721-30.

12. Metz JA, Preuss P, Komiyama N, et al. Discrimination between soft plaque and thrombus based on radiofrequency analysis of intravascular ultrasound. J Am Coll Cardiol 1996; 27(suppl A): 200 A (abstract).

13. Siegel RJ, Ariani M, Fishbein MC, et al. Histopathologic validation of angioscopy and intravascular ultrasound. Circulation 1991; 84: 109-17.

14. Di Mario C, Gorge G, Peters R, et al. Clinical application and image interpretation in intracoronary ultrasound. Eur Heart J 1998; 19: 207-29.

15. Glagov S, Weisenberg E, Zarins CK, et al. Compensatory enlargement of human atherosclerotic coronary arteries. N Engl Med 1986; 316: 1371-5.

16. Ge J, Erbel R, Zamorano J, et al. Coronary artery remodeling in atherosclerotic disease: an intravascular ultrasonic study in vivo. Coron Artery Dis 1993; 4 : 981-6.

17. Erbel R, Ge J, Bockisch A, et al. Value of intracoronary ultrasound and Doppler in the differentiation of angiographically normal coronary arteries: a prospective study in patients with angina pectoris. Eur Heart J 1996; 17: 880-9.

18. Abizaid AS, Mintz GS, Mehran R, et al. One year follow-up after percutaneous transluminal angioplasty was not performed based on intravascular ultrasound findings. Importance of lumen dimensions. Circulation 1999; 100: 256-61.

19. Mintz GS, Pichard AD, Kovach JA, et al. Impact of preintervention intravascular ultrasound imaging on transcatheter treatment strategies in coronary artery disease. Am J Cardiol 1994; 73: 423-30.

20. Gil R, Di Mario C, Prati F, et al. Influence of plaque composition on mechanisms of percutaneous transluminal coronary balloon angioplasty assessed by ultrasound imaging. Am Heart J 1996; 131: 591-7.

21. Waller BF. "Crackers, breakers, stretchers, drillers, scrapers, shavers, burners, welders and melters"- the future treatment of atherosclerotic coronary artery disease? A clinical-morphologic assessment. J Am Coll Cardiol 1989; 13: 969-87.

22. Honye J, Mahon DJ, Jain A, et al. Morphological effects of coronary balloon angioplasty in vivo assessed by intravascular ultrasound imaging. Circulation 1992; 85: 1012-25

23. Potkin BN, Keren G, Mintz GS, et al. Arterial responses to balloon coronary angioplasty: an intravascular ultrasound study. J Am Coll Cardiol 1992; 20: 942-51.

24. Dotter CT, Judkins MP. Transluminal treatment of arteriosclerotic obstruction. Description of a new technic and a preliminary report of its application. Circulation 1964; 30: 654-701.

25. Losordo DW, Rosenfield K, Pieczek A, Baker K, Harding M, Isner JM. How does angioplasty work? Serial analysis of human iliac arteries using intravascular ultrasound. Circulation 1992; 86: 1845-58.

26. van der Lugt A, Gussenhoven EJ, Stijnen T, et al. Comparison of intravascular ultrasonic findings after coronary balloon angioplasty evaluated in vitro with histology. Am J Cardiol 1995; 76: 661-6.

27. Stone GW, Hodgson JMcB, Goar FG, Frey A, Mudra H, for the CLOUT investigators. Improved procedural results of coronary angioplasty with intravascular ultrasound guided balloon sizing:The CLOUT Pilot Trial. Circulation 1997; 95: 2044-52.

28. Abizaid A, Pichard AD, Mintz GS, et al. Acute and long term results of an IVUS guided PTCA/provisional stent implantation strategy. Am J Cardiol 1999; 84 $1381-4$

29. Lee DY, Eigler N, Luo H, et al. Effect of intracoronary ultrasound imaging on clinical decision making. Am Heart J 1995; 129: 1084-93.

30. Fischman D, Leon MB, Baim D, et al. A randomized comparison of coronary stent placement and balloon angioplasty in the treatment of coronary disease. N Engl J Med 1994; 331: 496-501.

31. Serruys P, de Jaegere P, Kiemeneji F, et al. A comparison of ballon expandablestent implantation with balloon angioplasty in patients with coronary artery disease. N Engl J Med 1994; 331: 489-95.

32. Goldberg SL, Colombo A, Nakamura S, Almagor Y, Maiello L, Tobis JM. Benefit of intracoronary ultrasound in the deployment of Palmaz-Schatz stents. J Am Coll Cardiol 1994; 24: 996-1003.

33. Nakamura S, Colombo A, Gaglione A, et al. Intracoronary ultrasound observations during stent implantation. Circulation 1994; 89: 2026-34.

34. Kiemeneij F, Laarman G, Slagboom T. Mode of deployment of coronary PalmazSchatz stents after implantation with the stent delivery system: an intravascula ultrasound study. Am Heart J 1995; 129: 638-44.

35. Colombo A, Hall P, Nakamura S, et al. Intracoronary stenting withou anticoagulation accomplished with intravascular ultrasound guidance. Circulation 1995; 91: 1676-88.

36. Goods CM, Al-Shaibi KF, Yadav SS, et al. Utilization of the coronary balloonexpandable coil stent without anticoagulation or intravascular ultrasound Circulation 1996; 93: 1803-8.

37. Karrilion GJ, Morice MC, Benveniste E, et al. Intracoronary stent implantation without ultrasound guidance and with replacement of conventional anticoagulation by antiplatelet therapy. 30-day clinical outcome of the French Multicente Registry. Circulation 1996; 94: 1519-27.

38. Prati F, Gil R, Di Mario C, et al. Is quantitative angiography sufficient to guide stent implantation? A comparison with three-dimensional reconstruction of intracoronary ultrasound images. G Ital Cardiol 1997; 27: 328-36.

39. Allen KM, Undemir C, Schaknovich A, Moses J, Strin J, Kreps E. Is there need for intravascular ultrasound after high pressure dilatation of Palmaz-Schatz stents? J Am Coll Cardiol 1996; 27: 138A

40. Medeiros CR, Caixeta AM, Brito Jr FS, Perin MA, Rati MAN. Ganho luminal adicional com o uso do ultra-som intravascular como guia no implante de stents coronarianos (abstract). Rev Soc Cardiol ERJ 2000; 13 (Supl A): 89

41. Hoffmann R, Mintz GS, Pichard AD, Kent KM, Satler LF, Leon MB. Intimal hyperplasia thickness at follow-up is independent of stent size: a serial intravascular ultrasound study. Am J Cardiol 1998; 82: 1168-72.

42. Hodgson JMcB, Muller C, Roskamm H, et al. Improved acute outcomes during ultrasound-guided intervention achieved without increased device utilization or procedure time: findings from the strategy of ICUS-guided PTCA and stenting (SIPS) trial. J Am Coll Cardiol 1997; 29(Suppl A): 280A

43. Schiele F, Meneveau N, Vuillemenot A, et al. Impact of intravascular ultrasound guidance in stent deployment on 6-month restenosis rate: a multicenter, randomized study comparing two strategies - with and without intravascula ultrasound guidance. RESIST Study Group. REStenosis after Ivus guided Stenting. J Am Coll Cardiol 1998; 32: 320-8

44. de Jaegere P, Mudra H, Figulla H, et al. Intravascular ultrasound-guided optimized stent deployment. Immediate and 6 months clinical and angiographic results from the Multicenter Ultrasound Stenting in Coronaries Study (MUSIC Study). Eur Heart J 1998; 19: 1214-23.

45. Fitzgerald P, Hayase M, Mintz GS, et al. CRUISE: Can Routine Intravascular Ultrasound Influence Stent Expansion? Analysis of Outcomes. J Am Coll Cardiol 1998; 31: 396A.

46. Goldberg SL, Hall P, Nakamura S, et al. Is there a benefit from intravascula ultrasound when high pressure stent expansion is routinely performed prior to ultrasound imaging? J Am Coll Cardiol 1996; 27: 306A

47. Russo RJ, Nicosia A, Teirstein PS, Investigators AVID. Angiography versus intravascular ultrasound-directed stent placement. J Am Coll Cardiol 1997; 29 369A.

48. Kovach JA, Mintz GS, Pichard AD, et al. Sequential intravascular ultrasound characterization of the mechanisms of rotational atherectomy and adjunct balloon angioplasty. J Am Coll Cardiol 1993; 22: 1024-32. 
49. De Franco AC, Nissen SE, Tuzcu EM, Whitlow PL. Incremental value of intravascular ultrasound during rotational atherectomy. Cathet Cardiovasc Diagn 1996; Suppl: 23-33.

50. Braden GA, Herrington DM, Downes TR, Kutcher MA, Little WC. Qualitative and quantitative contrasts in the mechanisms of lumen enlargement by coronary balloon angioplasty and directional coronary atherectomy. J Am Coll Cardiol 1994; $23: 40-8$.

51. Marsico F, Kubica J, De Servi S, et al. Influence of plaque morphology on the mechanism of luminal enlargement after directional coronary atherectomy and balloon angioplasty. Br Heart J 1995; 74: 134-9.

52. Matar FA, Mintz GS, Pinnow E, et al. Multivariate predictors of intravascular ultrasound end points after directional coronary atherectomy. J Am Coll Cardiol 1995; $25: 318-24$

53. Baptista J, Di Mario C, Ozaki Y, et al. Impact of plaque morphology and composition on the mechanisms of lumen enlargement using intracoronary ultrasound and quantitative angiography after balloon angioplasty. Am J Cardiol 1996; 77: 115-21.

54. Simonton CA, Leon MB, Baim DS, et al. "Optimal" directional coronary atherectomy: final results of the Optimal Atherectomy Restenosis Study (OARS). Circulation 1998; 97: 332-9.

55. Topol E, Leya F, Pinkerton C, et al. A comparison of directional atherectomy with coronary angioplasty in patients with coronary artery disease. N Engl J Med 1993; 329: 221-7.
56. Adelman A, Cohen E, Kimball B, et al. A comparison of directional atherectomy with balloon angioplasty for lesions of the left anterior descending coronary artery. N Engl J Med 1993; 329: 228-33.

57. Mintz GS, Popma JJ, Pichard AD, et al. Intravascular ultrasound predictors of restenosis after percutaneous transcatheter coronary revascularization. J Am Coll Cardiol 1996; 27: 1678-87.

58. Mintz GS, Popma JJ, Pichard AD, et al. Arterial remodeling after coronary angioplasty: a serial intravascular ultrasound study. Circulation 1996; 94: 3543.

59. Painter JA, Mintz GS, Wong SC, et al. Serial intravascular ultrasound studies fail to show evidence of chronic Palmaz-Schatz stent recoil. Am J Cardiol 1995; 75: 398-400.

60. Dussaillant GR, Mintz GS, Pichard AD, et al. Small stent size and intimal hyperplasia contribute to restenosis: a volumetric intravascular ultrasound analysis. J Am Coll Cardiol 1995; 26: 720-4.

61. Di Mario C, von Birgelen C, Prati F, et al. Three dimensional reconstruction of cross sectional intracoronary ultrasound: clinical or research tool? Br Heart J 1995; 73: 26-32.

62. Stone GW, St Goar FG, Linnemeier TJ. Initial clinical experience with a novel low-profile integrated ultrasound-angioplasty catheter. Cathet Cardiovasc Diagn 1996; 38: 303-7.

63. Nissen SE, Ziada KM, Kapadia SR, Tuzcu EM. The current status of intravascular ultrasound imaging. Curr Probl Cardiol 1999; 24: 541-616.

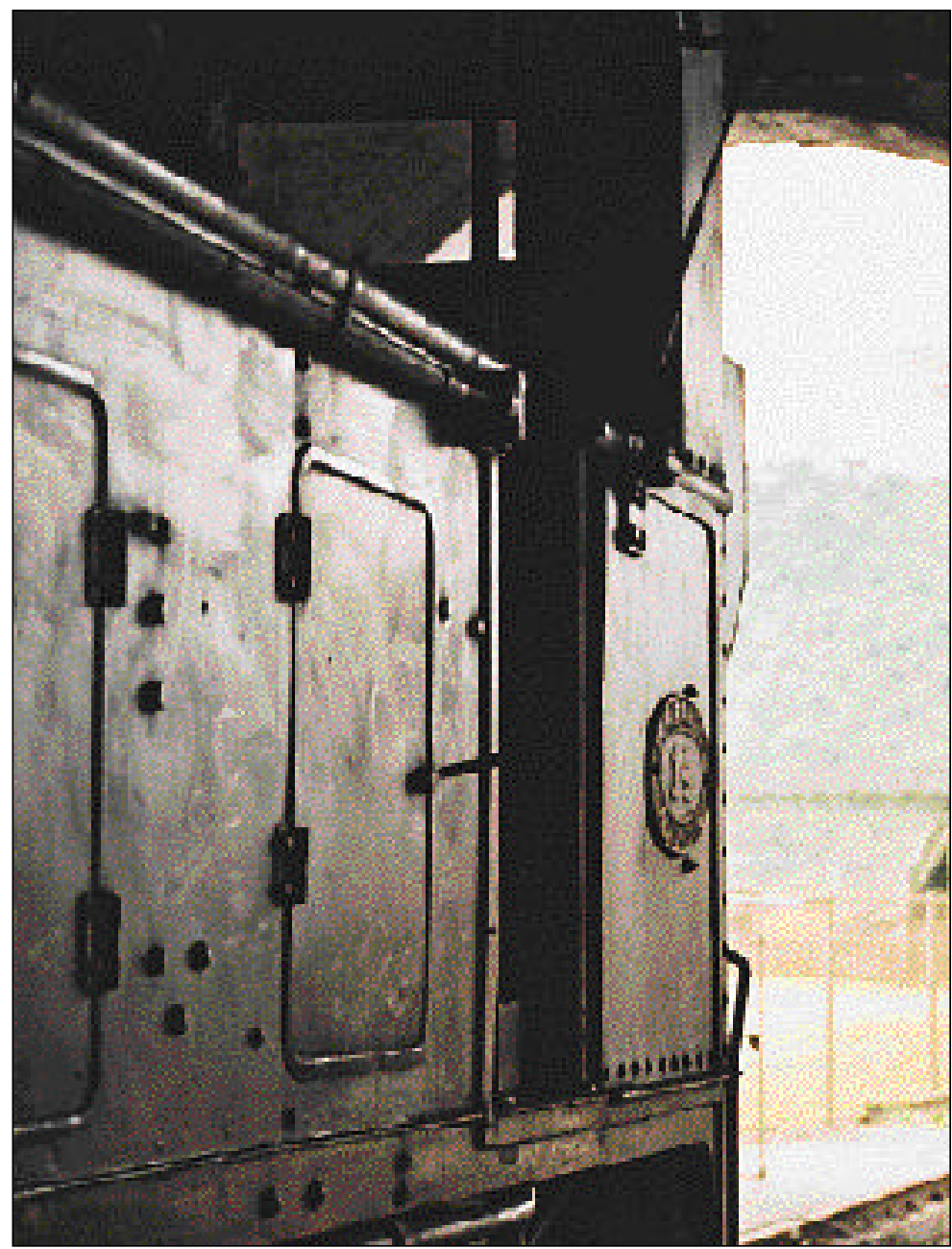

Paranapiacaba - SP

Márcio Tavares de Oliveira Jr.

Editor da Seção de Fotografias Artísticas: Cícero Piva de Albuquerque

Correspondência: InCor - Av. Dr. Enéas C. Aguiar, 44 - 05403-000 - São Paulo, SP - E-mail: delcicero@incor. usp.br 